\title{
Estratificação de Risco para Prevenção Primária de Morte Súbita Cardíaca em Cardiomiopatia Hipertrófica
}

\author{
Risk Stratification for Primary Prevention of Sudden Cardiac Death in Hypertrophic Cardiomyopathy \\ Styliani Vakrou, ${ }^{1}$ (10 Charalampos Vlachopoulos, ${ }^{1}$ Konstantinos A. Gatzoulis $^{1}$ \\ First University Department of Cardiology and Electrophysiology Laboratory, "Hippokration" General Hospital, National and Kapodistrian \\ University of Athens School of Medicine, ${ }^{1}$ Athens - Grécia
}

Lemos com grande interesse o artigo de Mattos et al. ${ }^{1}$ sobre estratificação de risco na cardiomiopatia hipertrófica $(\mathrm{CMH}){ }^{1}$ Os autores compararam as diretrizes de 2011 da American College of Cardiology Foundation/American Heart Association (ACCF/AHA) com as diretrizes de 2014 da European Society of Cardiology (ESC) em pacientes brasileiros com $\mathrm{CMH}$ e encontrou baixa concordância entre os dois sistemas. No total, 90 pacientes com $\mathrm{CMH}$ foram incluídos e 15 (17\%) deles receberam um cardioversor desfibrilador implantável (CDI). Dois (2\%) pacientes apresentaram choque apropriado, 6 (7\%) morte súbita cardíaca (MSC) e 6 (7\%) morte não cardíaca. De acordo com os critérios da ACCF/AHA de 2011, 43 (48\%) pacientes receberam recomendação de Classe Ila para CDI, 3 (3\%) receberam recomendação de Classe Ilb e 44 (49\%), Classe III. Se o escore de risco de MSC por MCH da ESC tivesse sido aplicado, 12 (14\%) pacientes teriam recebido recomendação de Classe lla para CDI (alto risco, $\geq 6 \%$ ), $11(12 \%)$ teriam recebido recomendação de Classe IIb (risco intermediário, $\geq 4 \%-<6 \%$ ) e 67 (74\%), Classe III (baixo risco, <4\%). O coeficiente kappa calculado $(0,355, p=0,0001)$ confirmou a baixa concordância entre as duas diretrizes. Especificamente falando, o modelo da ESC deixou desprotegidos todos os pacientes que apresentaram MSC ou abortaram MSC com apenas 2 deles (25\%) sendo classificados como Ilb. Os critérios da ACCF/AHA de 2011 recomendaram CDI (Classe Ila) para metade desses pacientes, com CDI sendo recomendado em muito mais pacientes do que os que realmente necessitavam. Os resultados deste estudo destacam a inadequação de estratégias precisas de estratificação de risco e contestam a tomada de decisão atual.

Esta não é a primeira vez que a precisão da predição de MSC na $\mathrm{CMH}$ provou ser insuficiente. ${ }^{2-4} \mathrm{~A} \mathrm{CMH}$ é a causa mais comum de MSC em jovens e a carga dessa perda devido à estratificação de risco não invasiva equivocada, por um lado, e a inserção desnecessária de um dispositivo, por outro, nos obrigam a melhorar esse quadro.

Nosso grupo propôs a contribuição adicional da estimulação ventricular programada (EVP) durante um estudo abrangente

\section{Palavras-chave}

Cardiomiopatia Hipertrófica; Morte Súbita Cardíaca; Desfibriladores Implantáveis; Prevenção e Controle; Síncope.

\section{Correspondência: Konstantinos A. Gatzoulis •}

National and Kapodistrian University of Athens School of Medicine - Cardiology

Vasilissis Sofias 114 Athens 11527 - Grécia

E-mail: kgatzoul@med.uoa.gr

Artigo recebido em 15/12/2020, revisado em 03/02/2021, aceito em 03/02/2021 de eletrofisiologia (EFS) nas estratégias de estratificação de risco atuais, a fim de abordar seu baixo desempenho ${ }^{4}$. A população do estudo incluiu 203 pacientes com $\mathrm{CMH}$, e como no estudo de Mattos et al., ${ }^{1}$ a maioria foi avaliada como risco baixo a intermediário para MSC (60\% apresentava um único fator de risco). Implantou-se CDI em 92 (45,3\%) pacientes, com ocorrência de desfecho primário (MSC e/ ou terapias apropriadas com CDI) em 20 pacientes (9,9\%). O achado importante do estudo foi que a EVP foi indutível em todos, exceto um paciente que apresentou o desfecho primário, ao passo que as diretrizes da AACF/AHA de 2011 e da ESC de 2014 classificaram erroneamente 3 e 9 pacientes, respectivamente. Em nossa população, as diretrizes de 2011 da ACCF/AHA teriam levado à implantação de 171 CDIs, enquanto as diretrizes da ESC teriam levado à implantação de 53, e, somente a EVP, em 79 pacientes. A combinação de cada uma dessas diretrizes com o protocolo EVP aumentaria esses números para 187 e 110 pacientes, respectivamente, sem perder nenhum desfecho primário. Principalmente em conjunto com as diretrizes da ESC, a sensibilidade e a especificidade ideais foram alcançadas da maneira mais econômica. Outra vantagem importante da EFS acabou sendo a caracterização adequada do mecanismo da síncope.

O uso da EVP na estratificação de risco de prevenção primária para MSC na CMHé a Classe III nas diretrizes europeias de 2014 e nas diretrizes da ACCF/AHA de 2011, com o principal argumento sendo os riscos de tal procedimento invasivo e seu custo, com base no nível não suportado de evidência C. No entanto, nosso método foi viável e seguro em todos os casos, enquanto a classificação incorreta ou a implantação inadequada de CDls são muito mais desoladoras e caras. O baixo desempenho das diretrizes da ESC e da ACCF/AHA pode ser devido a sua incapacidade de expressar o mecanismo exato de arritmogênese nesta doença. A ressonância magnética cardíaca (RMC) tem sido uma importante ferramenta na avaliação da $\mathrm{CMH}$, uma vez que a presença de fibrose de realce tardio com gadolínio é considerada um forte preditor independente para arritmias ventriculares malignas. ${ }^{5} \mathrm{O}$ uso de achados da RMC na estratificação de risco foi considerado nas diretrizes de 2020 da ACC/AHA.

Em conclusão, a identificação adequada de pacientes com maior risco de morte súbita indicados para a terapia com CDI, que salva vidas, com controle de tratamento excessivo, continua sendo o Santo Graal da CMH. Mattos et al.,1 apresentam evidências adicionais na literatura atual sobre as inadequações dos escores de risco e destacam a necessidade de introdução de critérios mais sensíveis e específicos. A estimulação ventricular programada pode ser uma nova ferramenta salvadora ao nosso arsenal contra a morte cardíaca súbita.

DOI: https://doi.org/10.36660/abc.20201339 


\section{Referências}

1. Mattos BP, Scolari FL, Garbin HI. Discrepancy between international guidelines on the criteria for primary prevention of sudden cardiac death in hypertrophic cardiomyopathy. Arq Bras Cardiol. 2020;115(2):197-204.

2. Wang J, Zhang Z, Li Y, Xu Y, Wan K, Chen Y. Variable and limited predictive value of the European Society of Cardiology hypertrophic cardiomyopathy sudden-death risk model: a meta-analysis. Can J Cardiol. 2019;35(2):1791-9.

3. Liu J, Wu G, Zhang C, Ruan J, Wang D, Zhang M et al. Improvement in sudden cardiac death risk prediction by the enhanced ACC/AHA strategy

\section{Carta-resposta}

A morte súbita cardíaca (MSC) é considerada a complicação mais dramática da cardiomiopatia hipertrófica $(\mathrm{CMH})$, com uma incidência anual estimada de 0,5-1\%. ${ }^{1}$ Ao longo dos anos, ACC/AHA e ESC publicaram diretrizes de consenso que apresentam diferentes abordagens para a estratificação de risco de MSC com base em preditores clínicos independentes. Apesar da acurácia relativamente baixa desses marcadores, as estratégias atuais teriam razoável poder discriminatório para o reconhecimento de pacientes de alto risco. No entanto, a avaliação prognóstica é um desafio na $\mathrm{CMH}$, principalmente em pacientes de risco baixo a moderado. A MSC pode surgir na ausência de fatores de risco conhecidos e algumas abordagens mostram resultados conflitantes em diferentes populações. ${ }^{2}$ Além disso, as discrepâncias metodológicas entre as diretrizes norte-americanas e europeias podem determinar níveis discordantes de recomendação em relação ao cardioversor desfibrilador implantável (CDI) na prevenção primária. ${ }^{3}$ Portanto, a estratificação de risco ainda é imprecisa e requer maior investigação.

O artigo de 2018 de Gatzoulis et al., ${ }^{4}$ reavalia o papel do estudo eletrofisiológico para a avaliação prognóstica em uma coorte unicêntrica de $\mathrm{CMH}$ de risco baixo a intermediário, estratificada de acordo com as diretrizes atuais. Os autores concluem que a estimulação ventricular programada (EVP) combinada com os modelos atuais acrescenta sensibilidade e valor preditivo negativo à estratificação de risco. ${ }^{4}$ Os resultados são interessantes, mas precisam ser confirmados em estudos prospectivos maiores. A EVP foi reduzida à classe III nas diretrizes atuais devido à baixa sensibilidade e aos possíveis riscos. Da mesma forma, o fracionamento dos eletrogramas do ventrículo direito tem sido relacionado à MSC, mas não foi assimilado devido à natureza invasiva do procedimento, o que pode afetar sua realização em alguns casos. ${ }^{5} \mathrm{~A}$ instabilidade elétrica é transitória na $\mathrm{CMH}$ e deve ser avaliada periodicamente assim como outros preditores in Chinese patients with hypertrophic cardiomyopathy. Heart Rhythm 2020;17(10):1658-63.

4. Gatzoulis KA, Georgopoulos S, Antoniou CK, Anastasakis A, Dilaveris P, Arsenos $P$ et al. Programmed ventricular stimulation predicts arrhythmic events and survival in hypertrophic cardiomyopathy. Int. J Cardiol. 2018;254:175-181.

5. Kariki O, Antoniou CK, Mavrogeni S, Gatzoulis KA. Updating the risk stratification for sudden cardiac death in cardiomyopathies: the evolving role of cardiac magnetic resonance imaging. An approach for the electrophysiologist. Diagnostics. 2020;10(8):541. com características dinâmicas. ${ }^{2,5}$ Deste modo, as diretrizes recomendam a reavaliação do risco de MSC durante o seguimento. Avaliação clínica não invasiva e de fácil aquisição favorece a abordagem de rotina do paciente.

Na última diretriz da ACC/AHA de 2020, foi introduzida um algoritmo com três novos preditores não invasivos: fibrose na forma de realce tardio através de ressonância magnética cardíaca com gadolínio, aneurisma apical do ventrículo esquerdo e fração de ejeção reduzida. ${ }^{1}$ Todos estes estão direta ou indiretamente relacionados a alterações na arquitetura miocárdica, principalmente a fibrose de substituição. Há uma tendência para a assimilação de novas e mais precisas tecnologias não invasivas para a avaliação prognóstica de rotina na $\mathrm{CMH}$ projetada para um futuro próximo. Talvez os protocolos invasivos entrem em desuso com base em avaliações de custo-efetividade. No entanto, a EVP pode ser aplicada em casos com estratificação de risco discordante entre as diretrizes em relação à recomendação de $\mathrm{CDI}$, podendo contribuir em casos com síncope cardíaca inexplicada.

Por fim, a avaliação do desarranjo celular com tensor de difusão por ressonância magnética cardíaca, genotipagem, perfil de microRNAs e outros biomarcadores promissores, bem como a avaliação não invasiva de isquemia miocárdica, poderão assegurar uma avaliação mais rigorosa do substrato anatômico e elétrico da doença. ${ }^{2,6}$ Porém, sua assimilação à prática clínica diária deve levar em consideração os recursos disponíveis localmente e representará um grande desafio. A busca por novos paradigmas na $\mathrm{CMH}$ está em curso e uma avaliação eletrofisiológica mais precisa dos mecanismos da MSC é necessária, podendo complementar ou mesmo suplantar a estratificação de risco de atual.

Beatriz Piva e Mattos Fernando Luís Scolari Henrique Ianhke Garbin 


\section{Referências}

1. Ommen SR, Mital S, Burke MA, Day SM, Deswal A, Elliott P, et al. 2020 AHA/ACC guideline for the diagnosis and treatment of patients with hypertrophic cardiomyopathy. Circulation 2020;142(25):e533-57. https://doi.org/10.1161/CIR.0000000000000937.

2. Pelliccia F, Gersh BJ, Camici PG. Gaps in evidence for risk stratification for sudden cardiac death in hypertrophic cardiomyopathy. Circulation 2021;143(2):101-3. https://doi.org/10.1161/CIRCULATIONAHA.120.051968.

3. Mattos BP, Scolari FL, Garbin HI. Discrepancy between international guidelines on the criteria for primary prevention of sudden cardiac death in hypertrophic cardiomyopathy. Arq Bras Cardiol 2020;115(2):197-204. https://doi.org/10.36660/abc.20190161.
4. Gatzoulis KA, Georgopoulos S, Antoniou CK, Anastasakis A, Dilaveris Arsenos P, et al. Programmed ventricular stimulation predicts arrhythmic events and survival in hypertrophic cardiomyopathy. Int J Cardiol 2018;254:175-81. https://doi.org/10.1016/j.ijcard.2017.10.033.

5. O'Mahony C, Elliott PM. Prevention of sudden cardiac death in hypertrophic cardiomyopathy. Heart 2014;100(3):254-60. https://doi.org/10.1136/ heartjnl-2012-301996.

6. Scolari FL, Faganello LS, Garbin HI, Piva e Mattos B, Biolo A. A systematic review of microRNAs in patients with hypertrophic cardiomyopathy. Int J Cardiol 2020;327:146-54. https://doi.org/10.1016/ j.ijcard.2020.11.004 